PROCEEDINGS OF THE

AMERICAN MATHEMATICAL SOCIETY

Volume 133, Number 6, Pages 1787-1796

S 0002-9939(04)07770-6

Article electronically published on November 19, 2004

\title{
ON A CUBIC-QUINTIC GINZBURG-LANDAU EQUATION WITH GLOBAL COUPLING
}

\author{
JUNCHENG WEI AND MATTHIAS WINTER \\ (Communicated by Carmen C. Chicone)
}

\begin{abstract}
We study standing wave solutions in a Ginzburg-Landau equation which consists of a cubic-quintic equation stabilized by global coupling

$$
A_{t}=\Delta A+\mu A+c A^{3}-A^{5}-k A\left(\int_{R^{n}} A^{2} d x\right) .
$$
\end{abstract}

We classify the existence and stability of all possible standing wave solutions.

\section{INTRODUCTION}

The study of pattern formation in various fields of science leads to systems with global coupling. Examples, some of which we will describe later, arise in fluid mechanics as well as chemistry or biology. In this paper, we consider pattern formation in a Ginzburg-Landau equation, where a cubic-quintic equation is stabilized by global coupling. This equation was suggested by Riecke in [13] and can be written as follows:

$$
A_{t}=\Delta A+\mu A+c|A|^{2} A-|A|^{4} A-k A \int_{R^{n}}|A|^{2} d x,
$$

where $A$ is a complex-valued function defined on $R^{n} \times(0, \infty), k>0$ and $c, \mu$ are real numbers.

To exclude the effects of dispersion, we consider the Ginzburg-Landau equation in its real-valued form. This means that we replace 1.1 by the following equation:

$$
A_{t}=\Delta A+\mu A+c A^{3}-A^{5}-k A \int_{R^{n}} A^{2} d x,
$$

where $A$ is a real-valued function defined on $R^{n} \times(0, \infty)$.

We note that the cubic-quintic equation arises by deriving the amplitude equations up to the fifth order. Very often it is not enough to expand just to the cubic term, and the quintic term becomes important. We refer to the interesting remarks in this direction given in Section 5 of [10]. There the authors consider a partial differential equation part of which is similar to the Swift-Hohenberg equation but which is of fourth order and also has a quadratic, symmetry breaking term. Then

Received by the editors November 5, 2002 and, in revised form, February 22, 2004

2000 Mathematics Subject Classification. Primary 35B35, 76E30; Secondary 35B40, 76 E06.

Key words and phrases. Cubic-quintic Ginzburg-Landau equation, stability, pattern formation. 
they show that it is important to include the quintic term into the amplitude equation to achieve good agreement of the profiles of localized patterns in the amplitude equation with the envelopes of the solutions of the partial differential equation.

We now describe some related work.

The amplitude equations (1.1) without the quintic term have been derived for hydrodynamic models in [7, 2], and 15] and for thermosolutal convection, rotating convection, and magnetoconvection, in 3 and 10 . The stability of various steady states to these amplitude equations was analyzed in [16].

In [14 an amplitude equation called a minimal model is given which is derived from the Navier-Stokes equations and consists of a cubic-quintic Ginzburg-Landau equation similar to (1.1). To exclude the effects of dispersion, in [12 the real-valued case is studied.

In this paper, we consider (1.2) and in particular study the effects of the quintic term on the existence and stability as well as the profile of standing waves.

We first consider the steady-state solutions of (1.2) which satisfy

$$
\Delta A+\mu A+c A^{3}-A^{5}-k A \int_{R^{n}} A^{2} d x=0 .
$$

A standing wave is a solution to (1.3) which satisfies $A>0, A \in H^{1}\left(R^{n}\right)$. Hence a standing wave satisfies the partial differential equation

$$
\Delta A-\hat{a} A+c A^{3}-A^{5}=0, \quad A>0, \quad A \in H^{1}\left(R^{n}\right),
$$

with the consistency condition

$$
\hat{a}=k \int_{R^{n}} A^{2} d x-\mu .
$$

Note that if $\hat{a}<0$, then there are no nontrivial solutions to (1.4). So we may write $\hat{a}=a^{2}$, where $a>0$. Moreover, it is obvious by the maximum principle that $c$ must be positive.

Now we rescale the equation (1.4). Set $A(x)=\beta \hat{A}(y)$, where $y=\alpha x$ and the positive constants $\alpha$ and $\beta$ will be chosen suitably below. Then (1.4) becomes

$$
\beta \alpha^{2} \Delta \hat{A}-a^{2} \beta \hat{A}+c \beta^{3} \hat{A}^{3}-\beta^{5} \hat{A}^{5}=0 .
$$

We choose

$$
a=\alpha, \quad c \beta^{3}=\beta \alpha^{2}, \quad \delta=\frac{\beta^{4}}{\alpha^{2}}
$$

which implies that

$$
a=\alpha=c \sqrt{\delta}, \quad \beta=\sqrt{c \delta} .
$$

This shows that $\hat{A}(y)=w_{\delta}(y)$, where $w_{\delta}$ is a solution of the equation

$$
\left\{\begin{array}{l}
\Delta w_{\delta}-w_{\delta}+w_{\delta}^{3}-\delta w_{\delta}^{5}=0 \\
w_{\delta}(|y|) \rightarrow 0 \text { as }|y| \rightarrow+\infty, \quad w_{\delta}(0)=\max _{y \in R^{n}} w_{\delta}(y) .
\end{array}\right.
$$

Thus (1.3) is reduced to (1.6) and the consistency condition

which is equivalent to

$$
a^{2}=k \int_{R^{n}} A^{2} d x-\mu
$$

$$
\beta(\delta):=\delta c^{2}-k c^{1-n} \delta^{\frac{2-n}{2}} \int_{R} w_{\delta}^{2}(y) d y=-\mu,
$$


where $w_{\delta}$ is given by (1.6). To summarize, a solution of (1.7) gives rise to a solution of (1.3) through the relation

$$
A_{\delta}(x)=\sqrt{c \delta} w_{\delta}(c \sqrt{\delta} x) .
$$

In Section 2, we will show that problem (1.6) has a unique ground-state solution if and only if $\delta \in\left(0, \frac{3}{16}\right)$.

The main result of this paper is the following theorem.

Theorem 1. (a) (Existence). Problem (1.2) has a standing wave steady-state solution if and only if

$$
\mu \geq-\max _{\delta \in\left(0, \frac{3}{16}\right)} \beta(\delta),
$$

where $\beta(\delta)$ is defined in (1.7). If (1.9) holds, then the steady-state solution $A_{\delta}$ of (1.2) is given by (1.7), (1.8), where $w_{\delta}$ is the unique solution of (1.6).

(b) (Stability). Let $A_{\delta}$ be given in (a). Then the stability of $A_{\delta}$ is determined by $\beta^{\prime}(\delta)$. More precisely, if $\beta^{\prime}(\delta)>0, A_{\delta}$ is linearly unstable. If $\beta^{\prime}(\delta)=0, A_{\delta}$ is neutrally stable. If $\beta^{\prime}(\delta)<0, A_{\delta}$ is linearly stable.

In the one-dimensional case, we can solve (1.7) explicitly. The result is stated in Section 4. Thus we give a complete answer to the existence and stability of standing wave solutions in $R^{1}$.

The organization of this paper is as follows: In Section 2, we study the parameterized ground state equation (1.6). In Section 3, we consider the stability of standing wave solutions. In Section 4 , we deal with the one-dimensional case.

\section{Parameterized ground states}

In this section, we study the parameterized ground state equation (1.6).

Observe that when $\delta=0, w_{\delta}$ exists and is unique if and only if $n \leq 3$. (Existence follows by a shooting method; see [8] and [11]. Radial symmetry is proved in [6] and uniqueness in $\left[9\right.$.) On the other hand, if $\delta>0$, we will show that $w_{\delta}$ exists for all $n$. To this end, we let

$$
g(v)=-v+v^{3}-\delta v^{5} .
$$

Note that for each $\delta<\frac{1}{4}$, there are exactly two positive roots to $g(v)=0$ given by

$$
t_{1}(\delta)=\sqrt{\frac{1-\sqrt{1-4 \delta}}{2 \delta}}<t_{2}(\delta)=\sqrt{\frac{1+\sqrt{1-4 \delta}}{2 \delta}} .
$$

Let

$$
c(\delta)=\int_{0}^{t_{2}(\delta)} g(s) d s .
$$

Then it is easy to see that for $\delta<\frac{3}{16}, c(\delta)>0$ and for $\delta>\frac{3}{16}, c(\delta)<0$.

We then have

Lemma 2. For each $\delta \in\left(0, \frac{3}{16}\right)$, the function $g(v)$ satisfies the following conditions: (g1) $g \in C^{3}(R, R), \quad g(0)=0, \quad g^{\prime}(0)=0$.

(g2) There exist $b, c>0$ such that $b<c$ and $g(b)=g(c)=0, g(v)>0$ in $(-\infty, 0) \cup(b, c)$, and $g(v)<0$ in $(0, b) \cup(c,+\infty)$.

(g3) $\int_{0}^{c} g(v) d v>0$. 
(g4) Let $\theta>b$ be the smallest positive number such that $G(u)=0$, where

$$
G(u)=\int_{0}^{u} g(s) d s .
$$

Let $\rho>b$ be the smallest number such that $\frac{g(u)}{u-\rho}$ is nonincreasing for $u \in(\rho, c)$. Then either

(i) $\theta \geq \rho$, or

(ii) $\theta<\rho$ with $K_{g}(u)$ nonincreasing in $(\theta, \rho), K_{g}(u) \geq K_{g}(\theta)$ for $u \in(b, \theta)$, and $K_{g}(u) \leq K_{g}(\rho)$ for $u \in(0, b) \cup(\rho, c)$, where

$$
K_{g}(u)=\frac{u g^{\prime}(u)}{g(u)} .
$$

Proof. The proof of this lemma is elementary, but we present it for the sake of completeness. The conditions (g1) $-(\mathrm{g} 3)$ are easy to verify. (Here $b=t_{1}(\delta), c=$ $t_{2}(\delta)$.) We only consider $(\mathrm{g} 4)$. We first compute $\rho$. By definition, there exists a $u_{0}>b$ such that

$$
g\left(u_{0}\right)=g^{\prime}\left(u_{0}\right)\left(u_{0}-\rho\right)
$$

and

$$
\left.\left(g(u)-g^{\prime}(u)(u-\rho)\right)^{\prime}\right|_{u=u_{0}}=0 .
$$

(2.5) implies that $g^{\prime \prime}\left(u_{0}\right)=0$, which gives $u_{0}=\sqrt{\frac{3}{10 \delta}}$. Now from (2.4) we get $\rho=u_{0}-\frac{g\left(u_{0}\right)}{g^{\prime}\left(u_{0}\right)}$. A straightforward calculation gives $\rho=\sqrt{\frac{3}{10 \delta}} \frac{24}{45-100 \delta}$. If $\rho \leq \theta$, we are done. (This is the case when $\delta$ is close to $\frac{3}{16}$.) Suppose that $\theta<\rho$. We need to calculate

$$
K_{g}(u)=\frac{-u+3 u^{3}-5 \delta u^{5}}{-u+u^{3}-\delta u^{5}}=1+\frac{2 u^{2}-4 \delta u^{4}}{-1+u^{2}-\delta u^{4}} .
$$

It is easy to compute that

$$
\frac{d}{d u}\left(K_{g}(u)\right)=\frac{4\left(-u+4 \delta u^{3}-\delta u^{5}\right)}{\left(-1+u^{2}-\delta u^{4}\right)^{2}} .
$$

Since $\delta<\frac{3}{16}$, it is easy to see that $\frac{d}{d u}\left(K_{g}(u)\right)<0$ for $u \in(b, c)$. This implies that $K_{g}(u)$ is nonincreasing in $(0, c)$. Moreover,

$$
K_{g}(u) \leq K_{g}(0)=1 \text {, for } u \in(0, b) .
$$

But

$$
K_{g}(\rho)=1+\frac{2 \rho^{2}\left(1-2 \delta \rho^{2}\right)}{-1+\rho^{2}-\delta \rho^{4}}>1 .
$$

Hence $K_{g}(u) \leq K_{g}(\rho)$ for $u \in(0, b) \cup(\rho, c)$. This shows that (g4) holds.

In the following lemma we state some important properties of $w_{\delta}$.

Lemma 3. If $\delta \in\left(0, \frac{3}{16}\right)$, then problem (1.6) has a unique solution $w_{\delta}$ that has the following properties.

(i) $w_{\delta} \in C^{\infty}\left(R^{n}\right)$.

(ii) $w_{\delta}>0$ is radially symmetric, and $w_{\delta}^{\prime}(r)<0$ for $r=|y| \neq 0$.

(iii) The first eigenvalue of the linear operator

$$
L_{\delta}=\Delta-1+3 w_{\delta}^{2}-5 \delta w_{\delta}^{4}: H^{2}\left(R^{n}\right) \rightarrow L^{2}\left(R^{n}\right),
$$


denoted by $\lambda_{1}=\lambda_{1}\left(L_{\delta}\right)$, is positive and simple; the corresponding eigenfunction $\Phi_{1}$ can be made positive and radially symmetric.

(iv) The second eigenvalue of $L_{\delta}$ is 0 , and the dimension is $n$. Namely, $\lambda_{2}\left(L_{\delta}\right)=$ 0 and

$$
\operatorname{Kernel}\left(\Delta-1+3 w_{\delta}^{2}-5 \delta w_{\delta}^{4}\right)=\operatorname{span}\left\{\frac{\partial w_{\delta}}{\partial y_{1}}, \ldots, \frac{\partial w_{\delta}}{\partial y_{n}}\right\} .
$$

(v) The operator $L_{\delta}: H_{r}^{2}\left(R^{n}\right)$ to $L_{r}^{2}\left(R^{n}\right)$ is invertible, where $H_{r}^{2}\left(R^{n}\right)=H^{2}\left(R^{n}\right)$ $\cap\{u=u(r)\}$ and $L_{r}^{2}\left(R^{n}\right)=L^{2}\left(R^{n}\right) \cap\{u=u(r)\}, r=|y|$.

Proof. The existence follows from a standard shooting method. (See 8, 11].) The radial symmetry follows from [6]. By Lemma 2 , for $\delta \in\left(0, \frac{3}{16}\right), g(v)=-v+v^{3}-\delta v^{5}$ satisfies conditions (g1)-(g4). Lemma 3 follows from Proposition 1.3 of [1]. (For uniqueness, see [5] and [11.)

The following lemma gives information about the dependence of $w_{\delta}$ on $\delta$ and provides some key identities.

Lemma 4. (1) $w_{\delta}$ is $C^{1}$ in $\delta$.

(2) As $\delta \rightarrow \frac{3}{16}, w_{\delta}(y) \rightarrow t_{2}\left(\frac{3}{16}\right)$ in $C_{\text {loc }}^{2}\left(R^{n}\right)$, where $t_{2}$ is defined in (2.2).

(3) If $n \leq 3$, then, as $\delta \rightarrow 0, w_{\delta} \rightarrow w_{0}$ in $C_{\text {loc }}^{2}\left(R^{n}\right)$, where $w_{0}$ is the unique solution of (1.6) with $\delta=0$.

(3) The following identities hold:

$$
\begin{gathered}
L_{\delta} w_{\delta}=2 w_{\delta}^{3}-4 \delta w_{\delta}^{5}, \\
L_{\delta} \frac{d w_{\delta}}{d \delta}=w_{\delta}^{5}, \\
L_{\delta}\left(y \cdot \nabla w_{\delta}\right)=2\left(w_{\delta}-w_{\delta}^{3}+\delta w_{\delta}^{5}\right), \\
L_{\delta}\left(\frac{1}{2} w_{\delta}+\delta \frac{d w_{\delta}}{d \delta}+\frac{1}{2} y \cdot \nabla w_{\delta}\right)=w_{\delta} .
\end{gathered}
$$

Proof. (1) follows from the uniqueness of $w_{\delta}$ given in Lemma 3

To prove (2), we note that $w_{\delta} \leq t_{2}(\delta)$ and hence, as $\delta \rightarrow \frac{3}{16}$, $w_{\delta}$ approaches in $C_{\text {loc }}^{2}\left(R^{n}\right)$ a solution of the equation

$$
\Delta u-u+u^{3}-\frac{3}{16} u^{5}=0, \quad u=u(|y|), \quad y \in R^{n},
$$

which admits only constant solutions. That constant must be $t_{2}\left(\frac{3}{16}\right)$ since $w_{\delta}(0) \rightarrow$ $t_{2}\left(\frac{3}{16}\right)$. This proves $(2)$.

The proof of (3) is similar: As $\delta \rightarrow 0, w_{\delta} \rightarrow w_{0}$ in $C_{l o c}^{2}\left(R^{n}\right)$, where $w_{0}$ is a solution of the problem

$$
\Delta w_{0}-w_{0}+w_{0}^{3}=0, w_{0}=w_{0}(|y|), w_{0}^{\prime}(|y|) \leq 0, y \in R^{n} .
$$

When $n \leq 3$, then by [9] there exists a unique solution to (2.12).

The first two identities (2.8) and (2.9) follow from direct computations and the third one (2.10) follows from Pohozaev's identity. (2.11) follows from (2.8) (2.10).

Now we consider the consistency condition

$$
\beta(\delta)=\delta c^{2}-k c^{1-n} \delta^{1-\frac{n}{2}} \int_{R^{n}} w_{\delta}^{2} d y=-\mu .
$$


We then have

Lemma 5. If $n=1$, then for any $\mu \geq 0$, problem (2.13) admits a solution.

If $n=2$, then problem (2.13) admits a solution for any $\mu \geq \mu_{0}$, where $\mu_{0}=$ $k c^{-1} \int_{R^{n}} w_{0}^{2}$.

If $n=3$, then problem (2.13) admits two solutions for $\mu \geq \hat{\mu}_{0}$, where $\hat{\mu}_{0}=$ $-\max _{\delta \in\left(0, \frac{3}{16}\right)} \beta(\delta)$.

Proof. Note that by Lemma 4 for all $n, \beta(\delta) \rightarrow-\infty$ as $\delta \rightarrow \frac{3}{16}$. On the other hand, we have as $\delta \rightarrow 0, \beta(\delta) \rightarrow 0$ if $n=1, \beta(\delta) \rightarrow-k c^{-1} \int_{R^{n}} w_{0}^{2}$ if $n=2, \beta(\delta) \rightarrow-\infty$ if $n=3$. Lemma 5 now follows from the mean-value theorem.

Remark 2.1. If $n=1$, we have a complete answer to (2.13). See Section 4 below.

\section{Stability analysis: Proof of Theorem [ 1}

In this section, we give necessary and sufficient conditions for the linear stability (or instability) of standing wave solutions.

We let

$$
A(x, t)=A_{\delta}(x)+\epsilon e^{\lambda t} \phi(x)
$$

where $A_{\delta}$ is given in (a) of Theorem 1. Substituting the above into (1.2) and collecting the $\epsilon$-terms, we obtain the following nonlocal eigenvalue problem:

$$
\Delta \phi-a^{2} \phi+3 c A_{\delta}^{2} \phi-5 A_{\delta}^{4} \phi-2 k A_{\delta} \int_{R^{n}} A_{\delta} \phi d x=\lambda \phi
$$

(using the notation in and after (1.4)). Substituting $A_{\delta}(x)=\sqrt{c \delta} w_{\delta}(c \sqrt{\delta} x), y=$ $c \sqrt{\delta} x$ into the above equation, we obtain

$$
\Delta \Phi-\Phi+3 w_{\delta}^{2} \Phi-5 \delta w_{\delta}^{4} \Phi-\gamma\left(w_{\delta} \int_{R^{n}}\left(w_{\delta} \Phi\right) d y\right)=\lambda \Phi,
$$

where

$$
\Phi(y)=\phi(x), \quad y=c \sqrt{\delta} x, \quad \gamma=2 k c^{-(n+1)} \delta^{-\frac{n}{2}} .
$$

Recall that (3.1) can be rewritten as

$$
L_{\delta} \Phi-\gamma\left(w_{\delta} \int_{R^{n}}\left(w_{\delta} \Phi d y\right)\right)=\lambda \Phi
$$

Note that $\lambda=0$ is an eigenvalue of (3.1) and the corresponding eigenfunction space is $\operatorname{Kernel}\left(L_{\delta}\right)$.

By arguments similar to [4, if $\lambda \neq 0$, we know that $\Phi \in H_{r}^{2}\left(R^{n}\right)$, i.e., the eigenfunctions (except for the kernel) are radially symmetric.

We first have

Lemma 6. $\lambda=0$ is an eigenvalue of (3.1) with a corresponding radially symmetric eigenfunction if and only if

$$
\gamma \int_{R^{n}} w_{\delta} L_{\delta}^{-1} w_{\delta} d y=1
$$

where $L_{\delta}^{-1}$ exists in $H_{r}^{2}\left(R^{n}\right)$ by Lemma 3 . 
Proof. Suppose $\lambda=0$. Then we have

$$
0=L_{\delta} \Phi-\gamma\left(\int_{R^{n}} w_{\delta} \Phi d y\right) w_{\delta}
$$

which implies that

$$
\Phi=\gamma\left(\int_{R^{n}} w_{\delta} \Phi d y\right) L_{\delta}^{-1} w_{\delta} .
$$

Multiplying (3.4) by $w_{\delta}$ and integrating, we obtain (3.3) since $\int_{R^{n}} w_{\delta} \Phi d y \neq 0$ (as otherwise $L_{\delta} \Phi=0$ and hence $\Phi=0$ by Lemma 3 ).

On the other hand, suppose that (3.3) holds true. Then for $\lambda$ an eigenfunction, $\Phi$ satisfies

$$
L_{\delta} \Phi-\frac{\int_{R^{n}} w_{\delta} \Phi d y}{\int_{R^{n}} w_{\delta}\left(L_{\delta}^{-1} w_{\delta}\right) d y} w_{\delta}=0
$$

and, applying the operator $L_{\delta}^{-1}$, it is easy to see that this is equivalent to

$$
\Phi=L_{\delta}^{-1} w_{\delta} .
$$

The following is the main result of this section.

Lemma 7. All eigenvalues of (3.1) are real and

(a) if $\gamma \int_{R^{n}} w_{\delta} L_{\delta}^{-1} w_{\delta} d y>1$, then for all eigenvalues of (3.1) we have $\lambda<0$;

(b) if $\gamma \int_{R^{n}} w_{\delta} L_{\delta}^{-1} w_{\delta} d y=1$, then for all eigenvalues of (3.1) we have $\lambda \leq 0$ and zero is an eigenvalue of (3.1) with eigenfunction $L_{\delta}^{-1} w_{\delta}$;

(c) if $\gamma \int_{R^{n}} w_{\delta} L_{\delta}^{-1} w_{\delta} d y<1$, then there exists an eigenvalue $\lambda_{0}>0$ of (3.1).

From Lemma 7 we see that $\gamma \int_{R^{n}} w_{\delta} L_{\delta}^{-1} w_{\delta} d y=1$ is the borderline case between stability and instability of (3.1).

Proof. It is easy to see that (3.1) is self-adjoint and hence all eigenvalues are real. Let $\lambda \geq 0$ be an eigenvalue of (3.1). We first claim that $\lambda \neq \lambda_{1}$, where $\lambda_{1}$ is the first eigenvalue of $L_{\delta}$ given by Lemma 3 . In fact, if $\lambda=\lambda_{1}$, then we have

$$
\gamma \int_{R^{n}} w_{\delta} \Phi d y \int_{R^{n}} w_{\delta} \Phi_{1} d y=0
$$

where $\Phi_{1}$ is the principal eigenfunction of $L_{\delta}$. By Lemma $3, \Phi_{1}>0$. This implies

$$
\int_{R^{n}} w_{\delta} \Phi d y=0
$$

and thus

$$
L_{\delta} \Phi=\lambda_{1} \Phi
$$

which implies that $\Phi=\Phi_{1}$. This is impossible since $\Phi_{1}>0$ (and thus $\int_{R^{n}} w_{\delta} \Phi_{1} d y>$ $0)$.

So $\lambda \neq \lambda_{1}$. By Lemma $3,\left(L_{\delta}-\lambda\right)^{-1}$ exists for $0<\lambda \neq \lambda_{1}$. Multiplying (3.1) by $w_{\delta}$ and integrating, it follows that $\lambda_{0}>0$ is an eigenvalue of (3.1) if and only if it satisfies the following algebraic equation:

$$
1-\gamma \int_{R^{n}}\left[\left(\left(L_{\delta}-\lambda_{0}\right)^{-1} w_{\delta}\right) w_{\delta}\right] d y=0 .
$$


(Here we have used the fact that $\int_{R^{n}} w_{\delta} \Phi d y=0$ would imply that $\Phi \equiv 0$.) Let

$$
\rho(t)=1-\gamma \int_{R^{n}}\left[\left(\left(L_{\delta}-t\right)^{-1} w_{\delta}\right) w_{\delta}\right] d y, \quad t \geq 0, \quad t \neq \lambda_{1} .
$$

Then $\rho(0)=1-\gamma \int_{R^{n}}\left(w_{\delta} L_{\delta}^{-1} w_{\delta}\right) d y$ and

$$
\rho^{\prime}(t)=-\gamma \int_{R^{n}}\left[\left(\left(L_{\delta}-t\right)^{-2} w_{\delta}\right) w_{\delta}\right] d y<0 .
$$

On the other hand,

$$
\begin{gathered}
\rho(t) \rightarrow-\infty \text { as } t \rightarrow \lambda_{1}, t<\lambda_{1}, \\
\rho(t) \rightarrow+\infty \text { as } t \rightarrow \lambda_{1}, t>\lambda_{1}, \\
\rho(t) \rightarrow 1 \text { as } t \rightarrow+\infty .
\end{gathered}
$$

Thus $\rho(t)>0$ for $t>\lambda_{1}$ and $\rho(t)$ has a (unique) zero in $\left(0, \lambda_{1}\right)$ if and only if $\rho(0)>0$, which is equivalent to $1-\gamma \int_{R^{n}}\left(w_{\delta} L_{\delta}^{-1} w_{\delta}\right) d y>0$.

This proves the lemma.

Let us now compute $\gamma \int_{R^{n}} w_{\delta} L_{\delta}^{-1} w_{\delta}$. We have by (2.11) of Lemma 4 ,

$$
\begin{gathered}
\gamma \int_{R^{n}} w_{\delta} L_{\delta}^{-1} w_{\delta}=2 k c^{-(n+1)} \delta^{-\frac{n}{2}} \int_{R^{n}} w_{\delta}\left(\frac{1}{2} w_{\delta}+\delta \frac{d w_{\delta}}{d \delta}+\frac{1}{2} y \cdot \nabla w_{\delta}\right) d y \\
=2 k c^{-(n+1)} \delta^{-\frac{n}{2}} \int_{R^{n}}\left(\frac{2-n}{4} w_{\delta}+\delta w_{\delta} \frac{d w_{\delta}}{d \delta}\right) d y .
\end{gathered}
$$

It is now easy to see that $1-\gamma \int_{R^{n}} w_{\delta} L_{\delta}^{-1} w_{\delta} d y=0$ if and only if $\frac{d \beta}{d \delta}=0$.

We arrive at the following corollary.

Corollary 8. Let $\delta$ be a solution of the consistency condition (2.13). Then

(a) if $\beta^{\prime}(\delta)>0$, problem (3.1) is unstable. Namely, there exists an eigenvalue $\lambda>0$ to (3.1).

(b) if $\beta^{\prime}(\delta)<0$, problem (3.1) is stable. Namely, for all nonzero eigenvalues $\lambda$ to (3.1), we have $\lambda<0$.

(c) if $\beta^{\prime}(\delta)=0$, problem (3.1) is neutrally stable. Namely, there exists an eigenvalue $\lambda=0$ to (3.1) with multiplicity 1 for radially symmetric eigenfunctions (and multiplicity $n+1$ for all eigenfunctions). All other eigenvalues are negative.

Theorem 1 now follows from Corollary 8.

\section{The ONE-DIMENSIONAL CASE}

In this section, we give a complete study of the consistency condition in the onedimensional case, thanks to the following explicit formula of $w_{\delta}$, which was derived in Section 5 of [10]:

$$
w_{\delta}(y)=\frac{\sqrt{b}}{\sqrt{\left(\frac{b-2}{4}+\cosh ^{2} y\right)}}, \quad \text { where } b=\frac{2}{\sqrt{1-\frac{16 \delta}{3}}} .
$$

Elementary computations show that

$$
\int_{R} w_{\delta}^{2}(y) d y=\int_{R} \frac{4 b}{(b-2)+4 \cosh ^{2} y} d y=\frac{b}{f} \log \left|\frac{b / 2+f}{b / 2-f}\right|,
$$


where $f>0, f^{2}=\frac{b^{2}}{4}-1$. Set $b=2 \cosh \theta$. Then $f=\sinh \theta$ and hence

$$
\int_{R} w_{\delta}^{2}(y) d y=4 \theta \operatorname{coth} \theta
$$

So (2.13) becomes

$$
\hat{\beta}(\theta):=\beta(\delta)=\frac{3}{16} \tanh ^{2}(\theta) c^{2}-\sqrt{3} k \theta=-\mu,
$$

which is a purely algebraic equation and can be solved explicitly.

This implies

Lemma 9. Suppose that $n=1$.

(a) If $\frac{12 k}{c^{2}}>1$, then for each fixed $\mu \geq 0$, there exists a unique solution $\delta$ such that $\beta(\delta)=0$. There is no solution if $\mu<0$. Moreover, we have $\beta^{\prime}(\delta)<0$.

(b) If $\frac{12 k}{c^{2}}=1$, then for each fixed $\mu \geq 0$, there exists a unique solution $\delta$ such that $\beta(\delta)=0$. There is no solution if $\mu<0$. Moreover, we have $\beta^{\prime}(\delta)=0$ for $\mu=\operatorname{arccosh}\left(\sqrt{\frac{3}{2}}\right)-\frac{\sqrt{3}}{4} \approx 0.225$.

(c) If $0<\frac{12 k}{c^{2}}<1$, there are exactly two solutions to $\beta^{\prime}(\delta)=0$, which are denoted as $0<\delta_{m}<\delta_{M}$. Let $\beta_{m}=\beta\left(\delta_{m}\right), \beta_{M}=\rho\left(\delta_{M}\right)$. Then $\beta(\delta)=0$ has a solution if and only if $\mu \geq-\max \left(\beta_{M}, 0\right)$.

If $\beta_{M}>0$, then for each $-\beta_{M}<\mu<0$, there are exactly two solutions to $\beta(\delta)=0$, denoted by $\delta_{1}<\delta_{2}$ and we have $\beta^{\prime}\left(\delta_{1}\right)>0, \beta^{\prime}\left(\delta_{2}\right)<0$. For each $0<\mu<-\beta_{m}$, there are exactly three solutions to $\beta(\delta)=0$, called $\delta_{1}<\delta_{2}<\delta_{3}$, and we have $\beta^{\prime}\left(\delta_{1}\right)<0, \beta^{\prime}\left(\delta_{2}\right)>0, \beta^{\prime}\left(\delta_{3}\right)<0$. If $\mu>-\beta_{m}$, then there is exactly one solution to $\beta(\delta)=0$ with $\beta^{\prime}(\delta)<0$.

The case $\beta_{M} \leq 0$ can be discussed similarly.

\section{ACKNOWLEDGMENTS}

The research of JW is supported by an Earmarked Grant from RGC of Hong Kong. MW thanks the Department of Mathematics at the Chinese University of Hong Kong for their kind hospitality.

\section{REFERENCES}

[1] P. Bates, E.N. Dancer and J. Shi, Multi-spike stationary solutions of the Cahn-Hilliard equation in higher-dimension and instability, Adv. Differential Equations 4 (1999), 1-69. MR 1667283 (99k:35097)

[2] P. Coullet and G. Iooss, Instabilities of one-dimensional cellular patterns, Phys. Rev. Lett. 64 (1990), 866-869. MF 1038266 (90m:82017)

[3] S.M. Cox and P.C. Matthews, New instabilities in two-dimensional rotating convection and magnetoconvection, Phys. D 149 (2001), 210-229. MR.1815525 (2002a:76068)

[4] E.N. Dancer, On stability and Hopf bifurcations for chemotaxis systems, IMS Workshop on Reaction-Diffusion Systems (Shatin, 1999), Methods Appl. Anal. 8 (2001), 245-256. MR 1904528 (2003k:35117)

[5] E.N. Dancer, A note on asymptotic uniqueness for some nonlinearities which change sign, Bull. Austral. Math. Soc. 61 (2000), 305-312. MR1748710(2001f:35122)

[6] B. Gidas, W.M. Ni, and L. Nirenberg, Symmetry of positive solutions of nonlinear elliptic equations in $R^{N}$, Adv. Math. Suppl. Stud. 7A (1981), 369-402. MR0634248 (84a:35083)

[7] S. Fauve, Pattern forming instabilities, pp. 387-491, in "Hydrodynamics and nonlinear instabilities", C. Godrèche and P. Manneville ed., Cambridge University Press, 1998. MR 1629959 (99i:76001) 
[8] R. Gardner and L.A. Peletier, The set of positive solutions of semilinear equations in large balls, Proc. Roy. Soc. Edinburgh Sect. A 104 (1986), 53-72. MR0877892 (88e:35063)

[9] M.K. Kwong, Uniqueness of positive solutions of $\Delta u-u+u^{p}=0$ in $R^{n}$, Arch. Rat. Mech. Anal. 105 (1989), 243-266. MR0969899 (90d:35015)

[10] P.C. Matthews and S.M. Cox, Pattern formation with a conservation law, Nonlinearity 13 (2000), 1293-1320. MR1767960 (2001b:76042)

[11] T. Ouyang and J. Shi, Exact multiplicity of positive solutions for a class of semilinear problems, J. Differential Equations 146 (1998), 121-156. MR1625731 (99f:35061)

[12] H. Herrero and H. Riecke, Bound pairs of fronts in a real Ginzburg-Landau equation coupled to a mean field, Phys. D 85 (1995), 79-92.

[13] H. Riecke, Localized structures in pattern-forming systems, in "Pattern formation in continuous and coupled systems" (Minneapolis, MN, 1998), 215-229, IMA Vol. Math. Appl. 115, Springer, New York, 1999. MR 1708870 (2000j:35259)

[14] H. Riecke and W.-J. Rappel, Coexisting pulses in a model for binary-mixture convection, Phys. Rev. Lett. 75 (1995), 4035-4038.

[15] M.I. Tribelsky and K. Tsuboi, New scenario for transition to turbulence?, Phys. Rev. Lett. 76 (1996), 1631-1634.

[16] J. Norbury, J. Wei and M. Winter, Existence and stability of singular patterns in a GinzburgLandau equation coupled with a mean field, Nonlinearity 15 (2002), 2077-2096. MR 1938482 (2003h:35024)

Department of Mathematics, The Chinese University of Hong Kong, Shatin, Hong KONG

E-mail address: wei@math.cuhk.edu.hk

Fachbereich Mathematik, Universität Stuttgart, D-70511 Stuttgart, Germany

E-mail address: winter@mathematik.uni-stuttgart.de 